\title{
Simplification of Moment Analysis Procedure for Kinetic Study of Chromatographic Behavior of Core-Shell Particles
}

\section{Kanji Miyabe}

Department of Chemistry, Faculty of Science, Rikkyo University, 3-34-1, Nishi-Ikebukuro, Toshima-ku, Tokyo 171-8501, Japan

E-mail: kmiyabe@rikkyo.ac.jp

\begin{abstract}
Moment analysis method for chromatographic behavior in core-shell columns was simplified. Mass transfer phenomena other than intra-stationary phase diffusion are analyzed with considering that the packing materials are spherical particles. The manner of intra-stationary phase diffusion is analyzed with assuming a hypothetical flat plate. For most core-shell particles commercially available, the geometry of spherical thin layer can be supposed as the hypothetical flat plate with a relative error less than ca. $2 \%$ because the thickness of the shell layer is sufficiently smaller than the diameter of whole particle. The supposition makes the moment analysis easier because the moment equations for flat plates are simpler than those strictly developed for core-shell particles. Some chromatographic data measured using a core-shell column were analyzed by the simple moment analysis method to confirm its usefulness. It is demonstrated that the method is effective for the preliminary study of mass transfer kinetics in core-shell columns.
\end{abstract}

Keywords: Moment theory, Simple moment analysis, Core-shell column, Spherical core-shell particle, Mass transfer kinetics, Intra-stationary phase diffusion 


\section{Introduction}

Core-shell particles have been developed for fast HPLC. ${ }^{1}$ They have a remarkably different structure in comparison with the conventional full-porous spherical particles. There is a spherical inert core at the central part of particles, which is surrounded by a porous thin layer. Many types of columns packed with core-shell particles are now commercially available. The diameter of whole particles and the thickness of the porous thin layer usually ranges from 2 to $4 \mu \mathrm{m}$ and 0.3 to $0.6 \mu \mathrm{m}$, respectively. ${ }^{1}$ The relatively large particle diameter enables a high flow rate convection of the mobile phase. The sufficient thin layer is effective to reduce the diffusion distance in the stationary phase. These contribute to the achievement of high-speed separation with a high efficiency.

Chromatographic behavior of various separation media has conventionally been analyzed by ordinary rate equations, ${ }^{2-5}$ e.g., van Deemter equation.

$$
H=A+\frac{B}{u}+C u
$$

where $A, B$, and $C$ are the coefficients of the A-, B-, and C-term, respectively, $u$ is the mobile phase flow velocity, and $H$ is the height equivalent to a theoretical plate (HETP). However, the structure of core-shell particles is not properly considered when the rate equations were developed. Although the shape of core-shell particles is spherical, their inner structure is quite different from conventional full-porous spherical particles. The contributions of external mass transfer and surface diffusion to $H$ is not considered in most of ordinary rate equations. ${ }^{6,7}$ The values of relating diffusivities and mass transfer coefficients cannot be quantitatively determined by the ordinary rate equations from the plot between $H$ and $u$. These problems suggest that chromatographic behavior of core-shell particles cannot sufficiently be analyzed by the ordinary rate equations.

One of alternatives is the moment theory, by which the first absolute $\left(\mu_{1 \mathrm{~A}}\right)$ and second central $\left(\mu_{2 \mathrm{C}}\right)$ moment of elution peaks are analyzed to derive information about retention equilibrium, mass transfer rate, and reaction kinetics in HPLC columns. ${ }^{8-17}$ Moment equations for various separation media having different structures have already been developed with appropriately considering their structures. ${ }^{18-22}$ There are also moment equations for core-shell particles. ${ }^{23,24}$ Two sets of moment equations having different mathematical formulas have been developed independently in two different manners.

Chromatographic behavior of core-shell particles has not usually been analyzed by the moment theory, in spite of its effectiveness in comparison with the ordinary rate theory. It may be a conceivable reason that the moment analysis procedure is mathematically complicated ${ }^{25-28}$ and that the moment equations strictly developed for core-shell particles are more complicated. ${ }^{23,24}$ A convenient procedure should be developed for analyzing HPLC behavior of core-shell particles on the basis of the moment theory. In a previous paper, ${ }^{29}$ the author developed a simple moment analysis procedure for HPLC behavior of full porous spherical particles and silica monoliths by introducing some appropriate suppositions and simplifications. 
Similarly, in this study, a simple procedure based on the approximation of the structure of core-shell particles as partially-porous flat plates was developed for the moment analysis of their chromatographic behavior. It was applied to some chromatographic data in a previous paper measured by using a core-shell column to demonstrate its usefulness.

\section{Theory}

Moment Equations for Full-Porous and Non-Porous Separation Media

For various separation media having different shapes, the moment equations for $\mu_{1 \mathrm{~A}}$ and $\mu_{2 \mathrm{C}}$ have been developed. ${ }^{18-20,22}$

$$
\begin{aligned}
& \mu_{1 A}=\frac{Z}{u}\left[1+\frac{\left(1-\varepsilon_{e}\right)}{\varepsilon_{e}}\left\{\varepsilon_{i}+\left(1-\varepsilon_{i}\right) K_{a}\right\}\right] \\
& \mu_{2 C}=\frac{Z}{u}\left[\frac{2 D_{L}}{u^{2}}\left[1+\frac{\left(1-\varepsilon_{e}\right)}{\varepsilon_{e}}\left\{\varepsilon_{i}+\left(1-\varepsilon_{i}\right) K_{a}\right\}\right]^{2}\right. \\
& \left.\quad+\frac{2\left(1-\varepsilon_{e}\right)}{\varepsilon_{e}}\left[\left(1-\varepsilon_{i}\right) \frac{K_{a}^{2}}{k_{r k}}+\left\{\frac{\xi^{2}}{\omega D_{e}}+\frac{\xi}{\theta k_{f}}\right\}\left\{\varepsilon_{i}+\left(1-\varepsilon_{i}\right) K_{a}\right\}^{2}\right]\right]
\end{aligned}
$$

where $\xi$ is the diffusion distance in the stationary phase, $\theta$ and $\omega$ are the numerical coefficients, respectively, $D_{\mathrm{e}}$, $k_{\mathrm{f}}, k_{\mathrm{rk}}$, and $D_{\mathrm{L}}$ are the effective intra-stationary phase diffusivity, the external mass transfer coefficient, the rate constant of chemical reaction, and the axial dispersion coefficient, respectively, $K_{\mathrm{a}}$ is the retention equilibrium constant, $\varepsilon_{\mathrm{i}}$ and $\varepsilon_{\mathrm{e}}$ are the internal and external porosity, respectively, $u$ is the mobile phase interstitial velocity, and $Z$ is the column length. The values of $\theta$ and $\omega$ for flat plates are 1 and 3, respectively. Equations (2) and

(3) do not represent the influence of sample injection on $\mu_{1 \mathrm{~A}}$ and $\mu_{2 \mathrm{C}}$ because it is negligible in usual HPLC experiments.

\section{Moment Equations for Core-Shell Particles}

Moment equations for core-shell particles has been developed..$^{23}$

$$
\begin{aligned}
& \mu_{1 A}=\frac{Z}{u}\left[1+\frac{\left(1-\varepsilon_{e}\right)\left(1-\varepsilon_{c}\right)}{\varepsilon_{e}}\left\{\varepsilon_{i}+\left(1-\varepsilon_{i}\right) K_{a}\right\}\right] \\
& \mu_{2 C}=\frac{2 Z}{\varepsilon_{e} u}\left(\delta_{a x}+\delta_{f}+\delta_{d}+\delta_{r k}\right) \\
& \delta_{a x}=\left(\frac{D_{L}}{\varepsilon_{e} u^{2}}\right)\left[\varepsilon_{e}+\left(1-\varepsilon_{e}\right)\left(1-\varepsilon_{c}\right)\left\{\varepsilon_{i}+\left(1-\varepsilon_{i}\right) K_{a}\right\}\right]^{2} \\
& \delta_{f}=\left(1-\varepsilon_{e}\right)\left(1-\varepsilon_{c}\right)^{2}\left(\frac{R_{2}}{3 k_{f}}\right)\left\{\varepsilon_{i}+\left(1-\varepsilon_{i}\right) K_{a}\right\}^{2}
\end{aligned}
$$




$$
\begin{aligned}
& \delta_{d}=\left(1-\varepsilon_{e}\right)\left(1-\varepsilon_{c}\right) \Omega^{*}\left(\frac{R_{2}^{2}}{15 D_{e}}\right)\left\{\varepsilon_{i}+\left(1-\varepsilon_{i}\right) K_{a}\right\}^{2} \\
& \delta_{r k}=\left(1-\varepsilon_{e}\right)\left(1-\varepsilon_{c}\right)\left(1-\varepsilon_{i}\right) \frac{K_{a}^{2}}{k_{r k}}
\end{aligned}
$$

where $\varepsilon_{\mathrm{c}}$ is the ratio of the volume of inert center core to that of whole particle and $\Omega^{*}$ is the parameter calculated by the related functions. ${ }^{23}$ Equations (6) - (9) represent the contributions $(\delta)$ of four kinetic processes in a column to $\mu_{2 \mathrm{C}}$.

\section{Approximation of Structural Characteristics of Core-Shell Particles}

Figure 1 indicates a possibility of approximation concerning the structure of core-shell particles. As shown in Fig. $1, R$ is the thickness of porous shell layer, $R_{1}$ and $R_{2}$ are the radius of central inert core and whole particle, respectively. The dominator of the ratio on the vertical axis is the true volume of the shell layer. The numerator represents the volume of a hypothetical flat plate, of which the thickness is equal to $R\left(=R_{2}-R_{1}\right)$ and the surface area is calculated from the average of $R_{1}$ and $R_{2}$. The vertical axis of Fig. 1 indicates the ratio of the volume of the hypothetical flat plate to that of the real shell layer. The horizontal axis shows the ratio of $R_{1}$ to $R_{2}$. The solid line in Fig. 1 illustrates the correlation between the two ratios on the vertical and horizontal axes. Figure 1 indicates that the difference between the volume of the hypothetical flat plate and that of the real shell layer decreases with a decrease in $R$.

Open circles in Fig. 1 correspond to real commercial products of core-shell particles. They are located in a limited range of $R_{1} / R_{2}$ from 0.60 to 0.90 . The relative error between the volume of the hypothetical flat plate and that of the real shell layer is less than ca. 2.0\%. This means that the real thin layer of core-shell particles can be assumed as a hypothetical flat plate, which has the same thickness $(R)$ and a corresponding surface area calculated from the average of $R_{1}$ and $R_{2}$. The moment analysis procedure for chromatographic behaviour of core-shell particles becomes easier because the moment equations for flat plates are simpler than those for core-shell particles.

It would be preferable that only the mass transfer in the porous shell layer is analysed by assuming the hypothetical porous flat plate. However, both the retention equilibrium and mass transfer kinetics other than intra-stationary phase diffusion in core-shell columns should be analyzed with considering that the shape of core-shell particles is spherical. For example, $D_{\mathrm{L}}$ and $k_{\mathrm{f}}$, which respectively correspond to axial dispersion and external mass transfer, depend on some experimental conditions, e.g., flow rate and viscosity of the mobile phase, size and shape of the stationary phase, temperature, and so on. It is required to take into account that the shape of core-shell particles is sphere when mass transfer phenomena in a column are analysed. The values of $D_{\mathrm{L}}$ and $k_{\mathrm{f}}$ for conventional HPLC columns packed with spherical particles are usually represented by Eqs. (S15) and (S17), respectively. 


\section{Simplification of Moment Analysis Procedure}

Many researchers of HPLC may consider that the moment analysis procedure for chromatographic behavior of core-shell particles is relatively complicated. ${ }^{25-28}$ Although the moment equation for $\mu_{2 \mathrm{C}}$ is complicated, Eqs. (4) - (9) have similar formulae including $K_{\mathrm{a}}, \varepsilon_{\mathrm{e}}, \varepsilon_{\mathrm{i}}$, and $\varepsilon_{\mathrm{c}}$, suggesting that a different moment analysis procedure can be developed without using $K_{\mathrm{a}}$ and $\varepsilon_{\mathrm{i}}$. The simplification of moment analysis procedure for chromatographic behavior of core-shell particles is explained in detail in Supporting Information.

The value of HETP $\left(H_{\text {total }}\right)$ is represented as follows.

$$
\begin{aligned}
H_{\text {total }}= & \frac{2 \varepsilon_{e} D_{L}}{u_{0}}+\frac{2 L^{2} \lambda^{2} u_{0}}{3\left(1-\varepsilon_{e}\right)\left(1-\varepsilon_{c}\right) D_{e}} \\
& +\frac{2 R_{2} \lambda^{2} u_{0}}{3\left(1-\varepsilon_{e}\right) k_{f}}+\frac{2 v^{2} u_{0}}{\left(1-\varepsilon_{e}\right)\left(1-\varepsilon_{c}\right)\left(1-\varepsilon_{i}\right) k_{r k}} \\
= & H_{a x}^{*}+H_{d}^{*}+H_{f}^{*}+H_{r k}^{*} \\
\lambda=1- & \frac{\varepsilon_{e} Z}{\mu_{1 A} u_{0}} \\
v=1- & \frac{\varepsilon_{e} Z+\left(1-\varepsilon_{e}\right)\left(1-\varepsilon_{c}\right) \varepsilon_{i} Z}{\mu_{1 A} u_{0}}
\end{aligned}
$$

where $L$ is the thickness of the hypothetical flat plate and $u_{0}$ is the superficial velocity of the mobile phase $\left(=\varepsilon_{\mathrm{e}} u\right)$. Equation (10) is modified as follows.

$$
\begin{aligned}
H_{\text {total }}= & \frac{2 \varepsilon_{e} \gamma_{1} D_{m}}{u_{0}}+2 \gamma_{2} d_{p}+\frac{2 L^{2} \lambda^{2} u_{0}}{3\left(1-\varepsilon_{e}\right)\left(1-\varepsilon_{c}\right) D_{e}} \\
& +\frac{2 R_{p} \lambda^{2}}{3\left(1-\varepsilon_{e}\right) \tau} u_{0}^{\frac{2}{3}}+\frac{2 v^{2} u_{0}}{\left(1-\varepsilon_{e}\right)\left(1-\varepsilon_{c}\right)\left(1-\varepsilon_{i}\right) k_{r k}}
\end{aligned}
$$

where $d_{\mathrm{p}}$ is the diameter of core-shell particles, $D_{\mathrm{m}}$ is the molecular diffusivity, and $\gamma_{1}$ and $\gamma_{2}$ are the geometrical coefficients. For conventional HPLC columns packed with spherical particles, $D_{\mathrm{L}}$ is usually accounted for by considering the contributions of molecular diffusion and eddy diffusion, as indicated in Eq. (S20). ${ }^{17}$ Consequently, $H_{\mathrm{ax}}{ }^{*}$ in Eq. (10) is rewritten as the first and second terms in the right hand side of Eq. (13), as indicated in Eq. (S21). The value of $k_{\mathrm{f}}$ was estimated by the Wilson-Geankoplis equation, as indicated in Eq. (S22). ${ }^{30,31}$ Similarly, $H_{\mathrm{f}}{ }^{*}$ in Eq. (10) is rewritten as the fourth term in the right hand side of Eq. (13), as indicated in Eq. (S25). On the other hand, $H_{\mathrm{d}}{ }^{*}$ and $H_{\mathrm{rk}}{ }^{*}$ in Eqs. (10) are respectively derived from Eqs. (S14) and (S18) by considering the definition of $H\left(=Z \mu_{2} \mathrm{~d} / \mu_{1 \mathrm{~A}}{ }^{2}\right)$.

Equation (13) is simply written as follows.

$$
H_{\text {total }}=\frac{B^{*}}{u_{0}}+A^{*}+C^{*} u_{0}+D^{*} u_{0}^{\frac{2}{3}}+E^{*} u_{0}
$$


The contribution of adsorption/desorption kinetics to $H_{\text {total }}\left(\mathrm{E}^{*}\right.$-term) is negligible in reversed-phase liquid chromatography (RPLC) because physical adsorption is fast enough. ${ }^{16}$ Equation (14) is rearranged as follows.

$$
H_{\text {total }}-D^{*} u_{0}^{\frac{2}{3}}=\frac{B^{*}}{u_{0}}+A^{*}+C^{*} u_{0}
$$

The formula of the right hand side of Eq. (15) is the same as that of the van Deemter equation, i.e., Eq. (1). However, $D_{\mathrm{e}}$ can quantitatively be calculated from the coefficient $C^{*}$ because its definition is clearly explained in Eq. (15). The contribution of $H_{\mathrm{f}}{ }^{*}\left(\mathrm{D}^{*}\right.$-term) to $H_{\text {total }}$ is also considered in Eq. (15). It is not considered in the van Deemter equation. Equation (1) cannot provide quantitative information about $D_{\mathrm{e}}$, either. Mass transfer kinetics in core-shell columns can be analyzed in more detail by the moment theory. Regarding the two points described above, Eq. (15) is different from Eq. (1).

\section{Experimental}

In a previous paper, ${ }^{32}$ chromatographic characteristics of core-shell columns were studied by analyzing the flow rate dependence of $H_{\text {total }}$. Basic information about the HPLC experiments is briefly explained in Supporting Information.

\section{Results and Discussion}

Flow rate dependence of HETP $(H)$ for Kal in RPLC system using the Halo column is shown in Fig. 2. Open circles represent the experimental data, of which correlation is indicated by the solid line. Figure 2a illustrates the correlation between the contributions of four mass transfer processes to $H_{\text {total }}$ and $u_{0}$. The results in Fig. 2a were obtained by the simple moment analysis procedure. As illustrated in Fig. 2b, similar results are obtained by the conventional moment analysis. The curved profile of the $\mathrm{B}^{*}$-term and that of the B-term are superposed with each other. A similar situation is also observed for the $\mathrm{A}^{*}$ - and A-term. On the other hand, there are differences between the profiles for the $\mathrm{C}^{*}$ - and C-term and for the $\mathrm{D}^{*}$ - and D-term, although the differences are not so significant.

Similarly, flow rate dependence of $H$ for Bra in the RPLC system using the same Halo column is shown in Fig. 3. Almost the same results are observed between Figures 3a and 3b. In Figs. 2a, 2b, 3a, and 3b, although it is unreasonable that the values of the $\mathrm{A}^{*}$ - and A-term are negative, it seems to be caused from the scattering of the experimental data illustrated by open circles. This has no influence on the theoretical validity of the simple moment analysis. Because the absolute values of the A*- and A-term are small, they provide little influence on the analytical results of $D_{\mathrm{e}}$ values described in the following.

In Figs. 2 and 3, dashed lines represent the flow rate dependence of the contribution of the C- and $\mathrm{C}^{*}$-terms to $H$. It is indicated that the steeper the slope of the dashed lines is, the larger the mass transfer 
resistance in the stationary phase is. In the previous paper, ${ }^{29}$ a simple moment analysis procedure was developed to study chromatographic properties of full porous spherical particles. Figures $1 \mathrm{a}$ and $1 \mathrm{~b}$ in the previous paper illustrate the analytical results of chromatographic behavior in RPLC systems, in which a column packed with full porous spherical particles, i.e., Mightysil, was used. Similarly, dashed lines in the figures represent the flow rate dependence of the contribution of the $\mathrm{C}^{*}$-term to $H$. The slope of the dashed lines in Figs. 2 and 3 in this study is comparable with that of the dashed lines in Figs. 1a and 1b in the previous paper. Chromatographic conditions are different between the two research works. For example, in the previous paper, ${ }^{29}$ propylbenzene and hexylbenzne were used as sample compounds, of which molecular weights are 120 and 162, respectively. On the other hand, kallidin and bradykinin were used in this study. Their molecular weights are respectively 1,188 and 1,060, which are about 6.5 - 9.9 times larger than those of propylbenzene and hexylbenzne. The contribution of the $\mathrm{C}$ - and $\mathrm{C}^{*}$-terms to $H$ increases with an increase in the molecular weight of sample compounds because the diffusive migration rate of small molecules is faster than that of large ones in the stationary phase. However, in spite of the large molecular weights of kallidin and bradykinin, the slope of the dashed lines for them in this study is almost of the same order of magnitude with that for propylbenzene and hexylbenzne in the previous paper. ${ }^{29}$ The results indicate the superior mass transfer characteristics of the superficial thin layer of core-shell particles as the stationary phase.

As indicated in Eqs. (13) - (15), the value of $D_{\mathrm{e}}$ can be calculated from the slope of the straight lines for the $\mathrm{C}^{*}$-term in Figs. 2a and 3a. The values of $D_{\mathrm{e}}$ were calculated as $5.5 \times 10^{-11}$ and $7.2 \times 10^{-11} \mathrm{~m}^{2} \mathrm{~s}^{-1}$ for Kal and Bra, respectively, from the coefficient $C^{*}$ in Eq. (15), which was derived by the simple moment analysis procedure. Similarly, the values of $D_{\mathrm{e}}$ were calculated as $3.3 \times 10^{-11}$ and $4.3 \times 10^{-11} \mathrm{~m}^{2} \mathrm{~s}^{-1}$ for Kal and Bra, respectively, from the slope of the straight lines for the C-term in Figs. $2 b$ and $3 b .^{23}$ They were obtained by the conventional moment analysis procedure. On the other hand, in the previous paper, ${ }^{32}$ the values of $D_{\mathrm{e}}$ were respectively reported as $3.0 \times 10^{-11}$ and $5.7 \times 10^{-11} \mathrm{~m}^{2} \mathrm{~s}^{-1}$ for Kal and Bra when the mobile phase composition was $\mathrm{H}_{2} \mathrm{O} / \mathrm{ACN} / \mathrm{TFA}=80 / 20 / 0.1$ (v/v\%), which was slightly different from that of the mobile phase used for measuring the $H-u_{0}$ plots in Figs. 2 and 3, i.e., $\mathrm{H}_{2} \mathrm{O} / \mathrm{ACN} / \mathrm{TFA}=82 / 18 / 0.1$ (v/v\%). Although the $D_{\mathrm{e}}$ values are not completely in agreement with each other, they are of the same order of magnitude. The simple moment analysis procedure is effective for the preliminary kinetic study of mass transfer phenomena in core-shell columns.

The simple moment analysis procedure requires only information about $\varepsilon_{\mathrm{e}}$ in Eqs. (13) - (15) when the $\mathrm{E}^{*}$-term is neglected. Different methods, such as, inverse size exclusion chromatography, ${ }^{33}$ Donan exclusion method, ${ }^{34}$ and total pore blocking, ${ }^{35}$ have been used for the experimental measurement of $\varepsilon_{\mathrm{e}}$, which range from ca. 0.35 to $0.43 .{ }^{36-41}$ It is also reported that $\varepsilon_{\mathrm{e}}$ of HPLC columns packed with spherical particles is ca. 0.40 $0.41 .^{2,17}$ It was tried to clarify how accurate results can be obtained by the simple moment analysis when the empirical $\varepsilon_{\mathrm{e}}$ values are used.

The dependence of $D_{\mathrm{e}}$ on $\varepsilon_{\mathrm{e}}$ is indicated in Fig. 4, in which $\varepsilon_{\mathrm{e}}$ was hypothetically changed from 0.35 to 0.45. Flow rate dependence of $H$ in Fig. 2 (Kal) and Fig. 3 (Bra) was analyzed by the simple moment analysis 
to derive $D_{\mathrm{e}}$ values. The values of $D_{\mathrm{e}}$ at $\varepsilon_{\mathrm{e}}=0.40$ are $-4.4 \%$ and $-5.9 \%$ smaller than those of $D_{\mathrm{e}}$ at $\varepsilon_{\mathrm{e}}=0.433$ for Kal and Bra, respectively. In Fig. 4, the relative error of $D_{\mathrm{e}}$ at $\varepsilon_{\mathrm{e}}$ between 0.35 and 0.45 against $D_{\mathrm{e}}$ at $\varepsilon_{\mathrm{e}}=$ 0.40 is $5.5-7.0 \%$ at maximum for Kal. Similarly, it is ca. $7.9-10.0 \%$ for Bra. More accurate results of moment analysis can be obtained when $\varepsilon_{\mathrm{e}}$ is experimentally measured.

\section{Conclusions}

Moment analysis procedure for studying the mass transfer kinetics in core-shell columns was simplified. The contributions of axial molecular diffusion, Eddy diffusion, and external mass transfer in a column to $\mu_{2 \mathrm{C}}$ are analyzed with considering that the shape of core-shell particles is spherical. On the other hand, only the contribution of intra-stationary phase diffusion to $\mu_{2 \mathrm{C}}$ is analyzed by assuming that the superficial porous thin layer can be approximated as a hypothetical porous flat plate. The moment analysis procedure for core-shell columns becomes easier by the supposition because the moment equations for a flat plate are simpler than those for core-shell particles, which are strictly developed.

The simple moment analysis procedure was applied to some chromatographic data measured by using a core-shell column (Halo column) in a previous study. Although the $D_{\mathrm{e}}$ values derived by the simple moment analysis were not completely in agreement with those derived by the conventional moment analysis using the strict moment equations for core-shell particles, they were of the same order of magnitude with each other. The same chromatographic data were analyzed by assuming a typical value of $\varepsilon_{\mathrm{e}}(=0.40)$. Resulting values of $D_{\mathrm{e}}$ were of the same order of magnitude with those obtained by using $\varepsilon_{\mathrm{e}}$ experimentally measured. In conclusion, the simple moment analysis is effective for the preliminary study of mass transfer kinetics in core-shell columns. The results of this study provide an opportunity of moment analysis concerning the mass transfer kinetics in core-shell columns to many researchers of HPLC because the simple procedure must be easy to use for them.

\section{Acknowledgements}

This work was supported by JSPS KAKENHI Grant Number 20K05570.

\section{Supporting Information}

A detailed explanation about the derivation of the moment equations and information about chromatographic conditions of HPLC experiments are provided. This material is available free of charge on the Web at http://www.jsac.or.jp/analsci/. 


\section{References}

1. V. González-Ruiz, A. I. Olives, and M. A. Martín, Trends Anal. Chem., 2015, 64, 17.

2. J. C. Giddings, "Dynamics of Chromatography”, 1965, Marcel Dekker, New York.

3. J. J. van Deemter, F. J. Zuiderweg, and A. Klinkenberg, Chem. Eng. Sci., 1956, 5, 271.

4. C. Horváth and H.-J. Lin, J. Chromatogr., 1976, 126, 401.

5. C. Horváth and H.-J. Lin, J. Chromatogr., 1978, 149, 43.

6. K. Miyabe and G. Guiochon, Adv. Chromatogr., 2000, 40, 1.

7. K. Miyabe and G. Guiochon, J. Chromatogr. A, 2010, 1217, 1713.

8. E. Kucera, J. Chromatogr., 1965, 19, 237.

9. M. Kubin, Collect. Czech Chem. Commun., 1965, 30, 2900.

10. E. Grushka, M. N. Myers, P. D. Schettler, and J. C. Giddings, Anal. Chem., 1969, 41, 889.

11. E. Grushka, J. Phys. Chem., 1972, 76, 2586.

12. M. Suzuki and J. M. Smith, Chem. Eng. Sci., 1971, 26, 221.

13. M. Suzuki, J. Chem. Eng. Jpn., 1973, 6, 540.

14. M. Suzuki and J. M. Smith, Adv. in Chromatogr., 1975, 13, 213.

15. D. M. Ruthven, "Principles of Adsorption \& Adsorption Processes", 1984, John Wiley and Sons, New York.

16. M. Suzuki, “Adsorption Engineering”, 1990, Kodansha/Elsevier, Tokyo/Amsterdam.

17. G. Guiochon, S. Golshan-Shirazi, and A M. Katti, "Fundamentals of Preparative and Nonlinear Chromatography", 1994, Academic Press, Boston.

18. K. Miyabe and G. Guiochon, J. Phys. Chem. B, 2002,106, 8898.

19. K. Miyabe, Anal. Chem., 2007, 79, 7457.

20. K. Miyabe, J. Chromatogr. A, 2008, 1183, 49.

21. K. Miyabe, J. Chromatogr. A, 2014, 1356, 171.

22. S. Fanali, P. R. Haddad, C. F. Poole, P. Schoenmakers, and D. Lloyd, "Liquid Chromatography: Fundamentals and Instrumentation", 2013, Elsevier, Amsterdam.

23. K. Miyabe, Anal. Sci., 2011, 27, 1007.

24. K. Kaczmarski and G. Guiochon, Anal. Chem., 2007, 79, 4648.

25. K. Miyabe and G. Guiochon, J. Sep. Sci., 2003, 26, 155.

26. K. Miyabe and G. Guiochon, J. Sep. Sci., 2004, 27, 853.

27. K. Miyabe, J. Sep. Sci., 2009, 32, 757.

28. K. Miyabe, Trends Anal. Chem., 2016, 81, 79.

29. K. Miyabe, Anal. Sci., 2021, 37, 593.

30. E. J. Wilson and C. J. Geankoplis, Ind. Eng. Chem. Fundam., 1966, 5, 9.

31. R. B. Bird, W. E. Stewart, and E. N. Lightfoot, “Transport Phenomena”, 2002, John Wiley and Sons, 
New York.

32. A. Cavazzini, F. Gritti, K. Kaczmarski, N. Marchetti, and G. Guiochon, Anal. Chem., 2007, 79, 5972.

33. M. Al-Bokari, D. Cherrak, and G. Guiochon, J. Chromatogr. A, 2002, 975, 275.

34. M. J. M. Wells and C. R. Clark, Anal. Chem., 1981, 53, 1341.

35. D. Cabooter, F. Lynen, P. Sandra, and G. Desmet, J. Chromatogr. A., 2007, 1157, 131.

36. J. Billen and G. Desmet, J. Chromatogr. A., 2007, 1168, 73.

37. F. Gritti and G. Guiochon, J. Chromatogr. A., 2012, 1221, 2.

38. J. S. Baker, J. C. Vinci, A. D. Moore, and L. A. Colón, J. Sep. Sci., 2010, 33, 2547.

39. K. Miyabe, H. Kobayashi, D. Tokuda, and N. Tanaka, J. Sep. Sci., 2006, 29, 2452.

40. K. Miyabe, Y. Sakai, M. Sarashina, and C. Yokokawa, Analyst, 2003, 128, 1425.

41. K. Miyabe, Y. Matsumoto, and G. Guiochon, Anal. Chem., 2007, 79, 1970. 


\section{Figure captions}

Fig. 1. Correlation between the ratio of the volume of hypothetical flat plate to that of real shell layer and the ratio of $R_{1}$ to $R_{2}$. Open circles represent information about representative commercial products of core-shell particles. Solid line illustrates the correlation between the two ratios on the vertical and horizontal axis.

Fig. 2. Dependence of $H$ and the contributions of four mass transfer processes to $H$ on $u_{0}$. Open circles represent original experimental data of kallidin, which were analyzed by (a) the simple and (b) conventional moment analysis procedure.

Fig. 3. Dependence of $H$ and the contributions of four mass transfer processes to $H$ on $u_{0}$. Open circles represent original experimental data of bradykinin, which were analyzed by (a) the simple and (b) conventional moment analysis procedure.

Fig. 4. $\quad$ Correlation between $D_{\mathrm{e}}$ and $\varepsilon_{\mathrm{e}}$. 
Figure 1

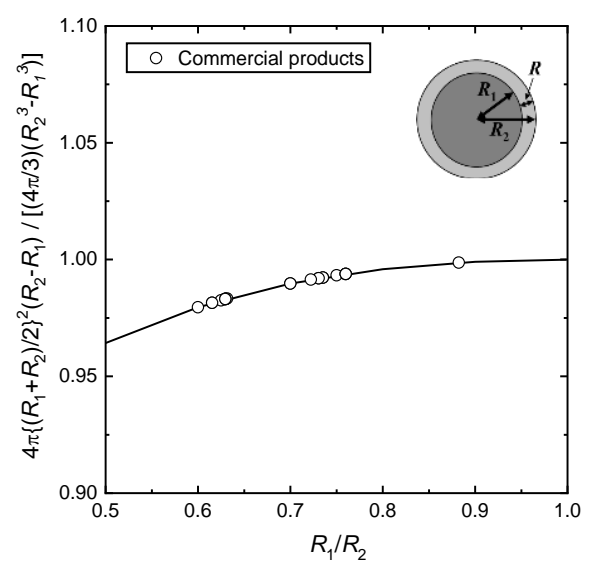


Figure 2a

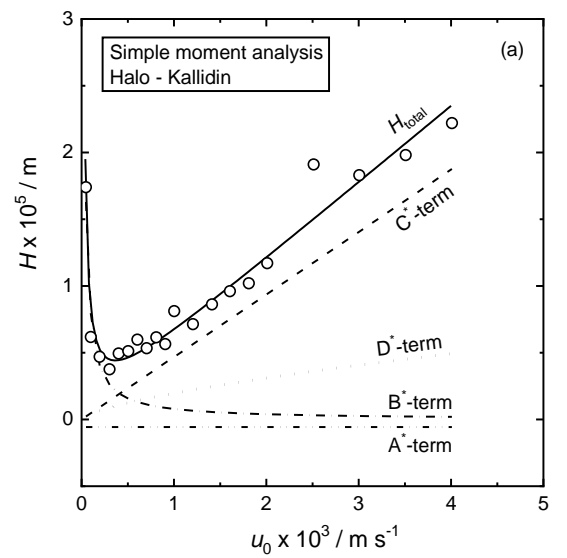


Figure $2 b$

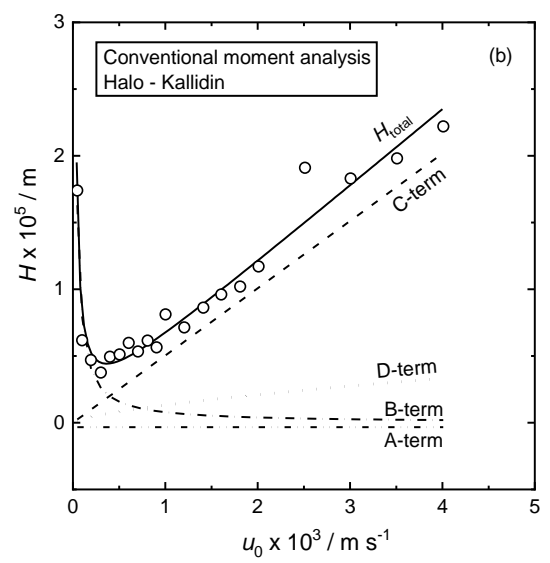


Figure 3a

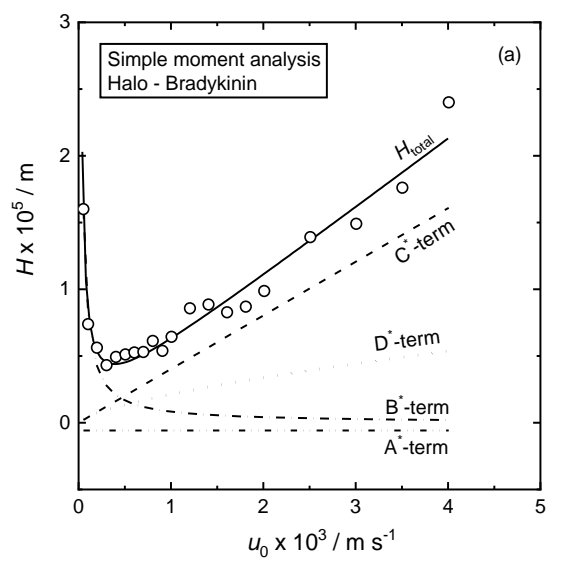


Figure $3 b$

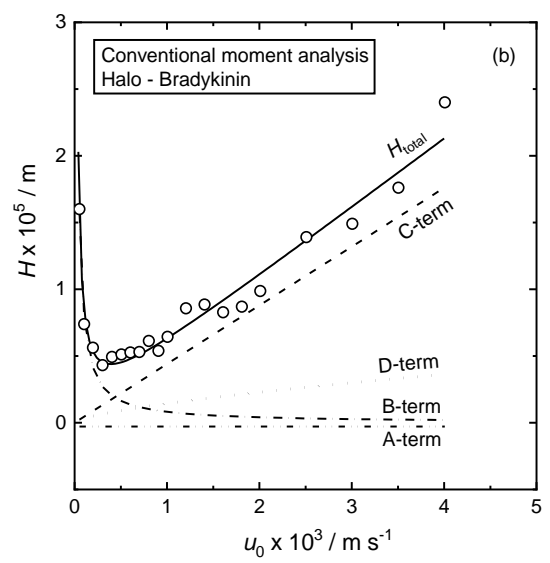


Figure 4

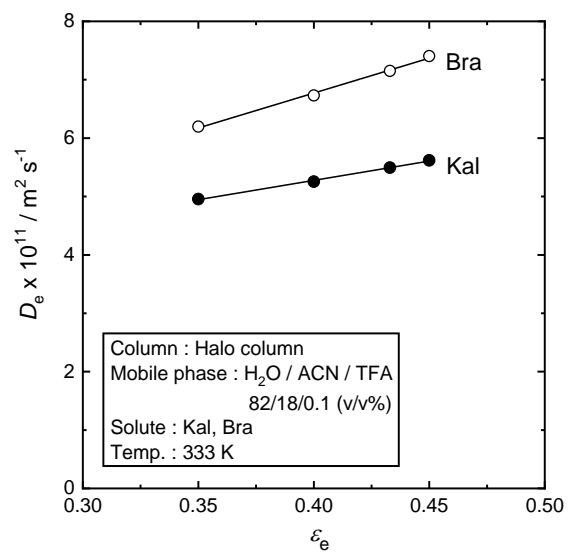


Graphical Index

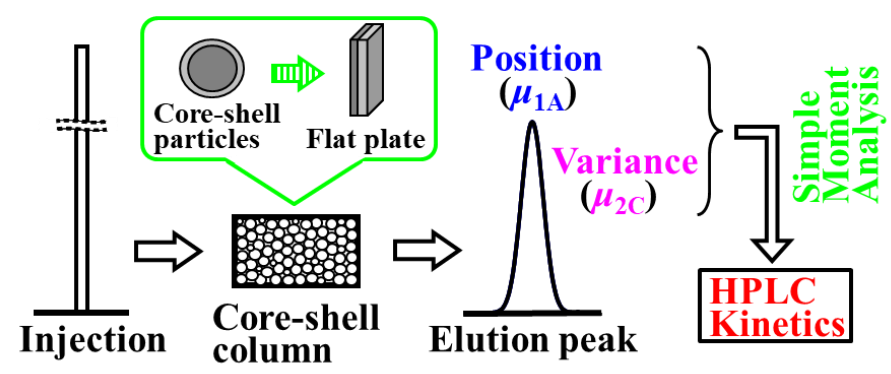

